\title{
Reallabore als Quelle für die Methodik transdisziplinären und transformativen Forschens - eine Einführung
}

\author{
Rico Defila \& Antonietta Di Giulio
}

\section{Worum es geht}

Den Hintergrund des vorliegenden Buchs bildet die Forschung in Reallaboren. Ein Reallabor ist ein Forschungsformat, in dem transdisziplinär geforscht wird und gleichzeitig ein expliziter transformativer Anspruch verfolgt wird.

Eine solche Forschung ist, unabhängig davon, wie Transdisziplinarität und Reallabore im Detail definiert werden (s. dazu auch Kapitel 2), in den Worten von Jürgen Mittelstraß ,weltlich“ (wie von ihm im Geleitwort zu diesem Buch auf den Punkt gebracht). Dies mag einer der Gründe sein dafür, dass ihre Ergebnisse innerhalb der Wissenschaft nicht vorbehaltlose Anerkennung finden, sondern auf eine gewisse Zurückhaltung, wenn nicht sogar Skepsis stoßen (für Rede und Gegenrede s. insbesondere Strohschneider 2014; Grunwald 2015; Schneidewind 2015; Rohe 2015; Krause 2017). Ein anderer Grund mag darin liegen, dass eine solche Forschung, mindestens ein Stück weit, eine ,Irritation“ des Wissenschaftssystems darstellt hinsichtlich der gesellschaftlichen Rolle von Wissenschaft, hinsichtlich des Stellenwerts gesellschaftlicher Ziele und Fragen in der Forschung sowie hinsichtlich der Partizipation außerwissenschaftlicher Akteure an Wissenschaft und Forschung. Das Forschungsformat Reallabor ist, so betrachtet, ein Beitrag zu dieser Debatte resp. eine Re-Aktualisierung dieser Debatte, indem neue Fragen gestellt werden bzw. bestehende Fragen neu gestellt werden. Ein dritter Grund für die Zurückhaltung mag darin liegen, dass die Standards zur Beurteilung guter Forschung nur in Teilen auf solche Forschung angewendet werden können. Es gilt also, diese Standards so weiter zu entwickeln, dass sie sowohl dazu dienlich sind, die Glaubwürdigkeit wissenschaftlicher Ergebnisse zu beurteilen, als auch den Besonderheiten dieser Forschung Rechnung tragen. Dazu möchte das vorliegende Buch beitragen. 
Konkret will das Buch einen Beitrag leisten zur Methodik transdisziplinärer, d. h. interdisziplinärer und partizipativer, Forschung, und zwar auch für solche, die nicht transformativ vorgeht (diese doppelte Ansprache ist im Folgenden durch Klammersetzung ausgedrückt: ,transdisziplinär (und transformativ)“). Zum einen werden ausgewählte Methoden der Wissenserzeugung, Wissensintegration und Transformation, die in konkreten Reallaboren entwickelt und umgesetzt wurden und die sich besonders bewährt haben, für die transdisziplinäre (und transformative) Forschung innerhalb und außerhalb von Reallaboren zur Verfügung gestellt. Zum anderen werden Kriterien, Fragen und Prinzipien mit Blick auf die Gestaltung transdisziplinärer (und transformativer) Forschung vorgestellt. Dabei wird auf die Phasen zu Beginn und in der Mitte eines Projekts fokussiert, d. h. die Schlussphase wird nicht beleuchtet. Anfang und Mitte sind sicher besonders bedeutsame Phasen transdisziplinärer (und transformativer) Forschung, diese Fokussierung hat aber auch ganz pragmatische Gründe: Zum Zeitpunkt des Verfassens der Buchbeiträge waren die Projekte, deren Erfahrungen hier reflektiert und aufbereitet vorliegen, noch am Laufen, d. h., es war gar nicht möglich, Einsichten und Erfahrungen, die sich auf die Schlussphase (bzw. auf die Zeit nach dem Ende der Projektförderung) beziehen, zu berücksichtigen.

Das Buch richtet sich in erster Linie an Personen, die transdisziplinär forschen (wollen) und dabei auch einen transformativen Anspruch verfolgen (wollen), und zwar innerhalb wie außerhalb des Formats Reallabor. Es richtet sich aber auch an Personen, die ohne einen solchen expliziten transformativen Anspruch transdisziplinär forschen (wollen).

\section{Transdisziplinarität, Transformation und Reallabore - und kein Zwang zur Ausrichtung auf Nachhaltigkeit}

Transdisziplinäre Forschung, transformative Forschung und Reallabore sind drei Begriffe, die in diesem Buch von zentraler Bedeutung sind, und die Frage, ob und inwieweit das Forschungsformat Reallabor an das Ziel einer Nachhaltigen Entwicklung gekoppelt ist, betrifft nicht nur dieses Forschungsformat selbst, sondern auch die Reichweite des vorliegenden Buchs. Sie sollen daher in diesem Kapitel in der gebotenen Kürze erörtert werden.

Transdisziplinäre Forschung wird hier akteurorientiert verstanden als Variante einer auf eine Synthese ausgerichteten interdisziplinären Forschung (s. auch Defila und Di Giulio 2018c). Bei dieser Variante beteiligen sich nicht nur Forscher(innen) aus verschiedenen wissenschaftlichen Disziplinen am Forschungsprozess, sondern zusätzlich auch Praxisakteure. Praxisakteure sind dabei substantiell am Projekt beteiligt, sei es als gleichberechtigte Mitglieder eines Projektteams oder als externe Beteiligte, die punktuell am Projekt mitwirken. Wenn Praxisakteure 
lediglich Untersuchungsgegenstand, Zielpublikum oder ,Echoraum“ der Forschung sind, handelt es sich gemäß diesem Verständnis nicht um eine transdisziplinäre Zusammenarbeit (zum Diskurs s. z. B. auch Burton et al. 2008; Defila und Di Giulio 1998; Klein 2014; Mobjörk 2010).

Für den Begriff der transformativen Forschung wiederum ist, mindestens im deutschen Diskurs, das Hauptgutachten „Welt im Wandel: Gesellschaftsvertrag für eine Große Transformation" des Wissenschaftlichen Beirats der Bundesregierung Globale Umweltveränderungen (WBGU) aus dem Jahr 2011 maßgebend. In diesem wird die Forderung nach einer Forschung formuliert, die gesellschaftliche Veränderungen nicht nur untersucht und entsprechendes System-, Ziel- und Transformationswissen zur Verfügung stellt (CASS und ProClim- 1997), sondern auch gezielt auf eine gesellschaftliche Transformation Richtung Nachhaltigkeit hinwirkt und in diesem Sinne transformativ tätig wird (WBGU 2011 und in der Folge z. B. Schneidewind und Singer-Brodowski 2014; s. aber z. B. auch Bäckstrand 2003; Mielke et al. 2016; Otero et al. 2017; Scholz 2017). In der Debatte um die gesellschaftliche Wirkung, die Forschung haben kann und soll, werden transformative Forschung, partizipative Forschung und transdisziplinäre Forschung argumentativ zusammengeschlossen, ausgehend von der Annahme, dass die Partizipation von Praxisakteuren an Forschung deren gesellschaftliche Relevanz und Wirkung verstärkt. International wird diese Debatte geführt z. B. im Kontext von Forschungsnetzwerken wie der „Great Transition Initiative“ (GTN) oder der „Sustainable Consumption Research and Action Initiative“ (SCORAI).

Das Forschungsformat Reallabor reiht sich hier ein. Es stellt einen Versuch dar, transformative Forschung in ein spezifisches Forschungsformat zu kleiden (für einen Überblick u. a. auch über die internationale Landschaft s. z. B. TATuPSchwerpunkt 2016; Schäpke et al. 2017; BGL-Themenheft 2017; GAIA Special Issue 2018). Reallabore verfolgen damit eine doppelte Zielsetzung, die Produktion von Erkenntnissen und neuem Wissen (Forschungsziele) und das Anstoßen von Transformationsprozessen (Praxisziele); zu den Merkmalen von Reallaboren sei hier auf Beecroft et al. (2018) verwiesen, die diese Zieldimensionen entfalten und als dritte Zieldimension Bildungsziele vorschlagen, sowie auf Arnold und Piontek (2018).

Transformative Forschung im Allgemeinen und das Forschungsformat Reallabor im Speziellen werden oftmals implizit oder explizit gleichgesetzt mit einer Forschung, die zu einer Nachhaltigen Entwicklung beitragen will (bzw. soll). Das ist, zumindest im deutschen Sprachraum, sicher der Entstehungsgeschichte geschuldet. Es gibt jedoch keinen zwingenden im Forschungsformat angelegten Grund, dass dem so ist, d. h. transformative Forschung im Allgemeinen und das Format Reallabor im Speziellen können auch andere Ziele als das Ziel einer Nachhaltigen Entwicklung anvisieren. Aus zwei Gründen kann dies aber auch kein beliebiges Ziel sein: Erstens ist eine Forschung, die aktiv und gezielt eine gesellschaftliche 
Veränderung anstoßen will, in besonderer Weise begründungs- und rechenschaftspflichtig, was die Ziele einer solchen Transformation betrifft. Zweitens ist Forschung, soweit sie aus öffentlichen Mitteln finanziert wird, dieser Öffentlichkeit gegenüber verpflichtet (wie relevant dies ist angesichts des Verhältnisses des Umfangs an Forschungsmitteln, der seitens der öffentlichen Hand zur Verfügung gestellt wird, im Vergleich zu dem, der durch wirtschaftliche und private Akteure zur Verfügung gestellt wird, sei hier dahingestellt).

Nachhaltigkeit bzw. eine Nachhaltige Entwicklung erfüllt als gesellschaftliches Ziel gewisse Kriterien, die sich isolieren lassen, die aber kumulativ gelten. Für den Kontext, um den es an dieser Stelle geht, sind dies insbesondere folgende: ${ }^{1}$

- Es handelt sich um ein Ziel, das in gesellschaftspolitischer Hinsicht legitimiert ist, d. h., es ist ein gesellschaftlich legitimiertes Ziel.

- Es handelt sich um ein Ziel, für das sich überzeugende ethische Argumente vorbringen lassen, d. h., es ist ein Ziel, das ethisch gut begründet ist.

- Es handelt sich um ein Ziel, das nicht auf einzelne gesellschaftliche Gruppen ausgerichtet ist, sondern auf die ganze Gesellschaft, d. h., es ist ein Ziel, das aufs Gemeinwohl (in Gegenwart wie Zukunft) orientiert ist.

Diese Kriterien stellen verallgemeinerbare Anforderungen für transformative Forschung dar: Transformative Forschung im Allgemeinen und das Format Reallabor im Speziellen sollten sich auf Ziele ausrichten, die diese drei Kriterien kumulativ erfüllen, die also gesellschaftlich legitimiert, ethisch gut begründet und gemeinwohlorientiert sind. ${ }^{2}$ Ein solcher Ansatz erlaubt es, das Forschungsformat Reallabor aus dem Kontext der Nachhaltigkeit herauszulösen und auch anderen Kontexten zugänglich $\mathrm{zu}$ machen, ohne das Format jedoch für beliebige transformative Zwecke zu öffnen. ${ }^{3}$

Damit richtet sich das vorliegende Buch auch an Personen, die zwar transformativ forschen (möchten), dies aber nicht mit Blick auf eine Nachhaltige Entwicklung tun, sondern mit Blick auf ein anderes gesellschaftliches Ziel.

1 Damit ist nicht gemeint, dass konkrete Aktivitäten, die unter dem Titel der Nachhaltigkeit geplant und/oder umgesetzt werden, diese Kriterien erfüllen, sondern lediglich, dass das übergreifende Ziel einer Nachhaltigen Entwicklung diese Kriterien erfüllt.

2 Krohn, Grunwald und Ukowitz (2017) streichen die Bedeutung der Gemeinwohlorientierung ebenfalls hervor (jedoch bezogen auf transdisziplinäre Forschung, die sich am Leitbild der Nachhaltigkeit orientiert).

3 Was jedoch nicht davon entbindet, die Legitimität von Forschenden, transformativ tätig zu werden, nicht als selbstverständlich gegeben anzunehmen, sondern vielmehr kritisch unter die Lupe zu nehmen; s. dazu Defila und Di Giulio (2018a) sowie Eckart et al. (2018). 


\section{In welchem Kontext und wie dieses Buch entstand}

Das Ministerium für Wissenschaft, Forschung und Kunst Baden-Württemberg (MWK) fördert(e) seit 2015 in zwei Förderlinien, den Förderlinien „Reallabore“ und „Reallabore Stadt“, insgesamt 14 Reallabore, von denen die meisten zum Zeitpunkt der Drucklegung dieses Buchs noch liefen, während bei einigen aus der ersten Förderlinie die Förderung bereits beendet war (die vom MWK geförderten Reallabore werden im Folgenden als „BaWü-Labs“ bezeichnet). ${ }^{4}$ Zusätzlich zu den 14 BaWü-Labs wurden zwei Begleitforschungs-Projekte gefördert (s. Schäpke et al. 2016). Für eines der beiden Begleitforschungs-Projekte, die zum Zeitpunkt der Drucklegung dieses Buchs beide ebenfalls noch liefen, zeichnen die Autorin und der Autor dieses Beitrags verantwortlich (im Folgenden als „BFBasel" bezeichnet; s. auch Defila und Di Giulio 2018b).

Das vorliegende Buch ist ein Ergebnis, das im Rahmen der Aktivitäten der BFBasel entstand. Die BF-Basel richtet(e) in regelmäßigen Abständen (ca. alle neun Monate) zweitägige Workshops aus, die darauf abziel(t)en, den BaWü-Labs einen Raum und eine methodische Struktur für die gemeinsame Reflexion, die wechselseitige Beratung, das gegenseitige Lernen und die syntheseorientierte Zusammenarbeit zur Verfügung zu stellen (s. Abb. 1). Die BF-Basel konzipiert(e) diese Workshops basierend auf theoretischen und methodischen Ansätzen einer guten inter- und transdisziplinären Forschungspraxis, sie moderiert(e) die Workshops und sorgt(e) für die Ergebnissicherung, während die Wahl der Themen für die einzelnen Workshops in Abstimmung mit den BaWü-Labs erfolgt(e). Das übergeordnete Format für diese Workshops ist das „Diskussionsforum“ (die Workshops waren bzw. sind also „Treffen des Diskussionsforums“).

Nur die Themen in den Blick nehmend, die sich auf das vorliegende Buch beziehen, wurden an den ersten drei Treffen des Diskussionsforums Ziel, Inhalt und Aufbau besprochen (Oktober 2015, Juli 2016, Februar 2017), und am Ende des vierten Treffens (Dezember 2017) fand das interne Review der Beiträge in Teil 1 des Buchs statt (für das interne Review der Beiträge in Teil 2 gab es ein gesondertes Treffen im November 2017). An den Diskussionen über Ziel, Inhalt und Aufbau des Buchs beteiligten sich damit auch Personen, die nicht in der Liste der Autor(inn)en erscheinen, und es flossen Erfahrungen und Überlegungen auch aus BaWü-Labs ein, die keinen Beitrag zum Buch verfassten (diese Erfahrungen finden sich auch in Form von Illustrations-Beispielen in den Beiträgen in Teil 1).

4 Für Informationen zu den Förderlinien „Reallabore“ und „Reallabore Stadt“ sowie eine Kurzbeschreibung aller geförderten BaWü-Labs siehe https://mwk.baden-wuerttem berg.de/de/forschung/forschungspolitik/wissenschaft-fuer-nachhaltigkeit/reallabore/ (zugegriffen am 14.02.2018). 


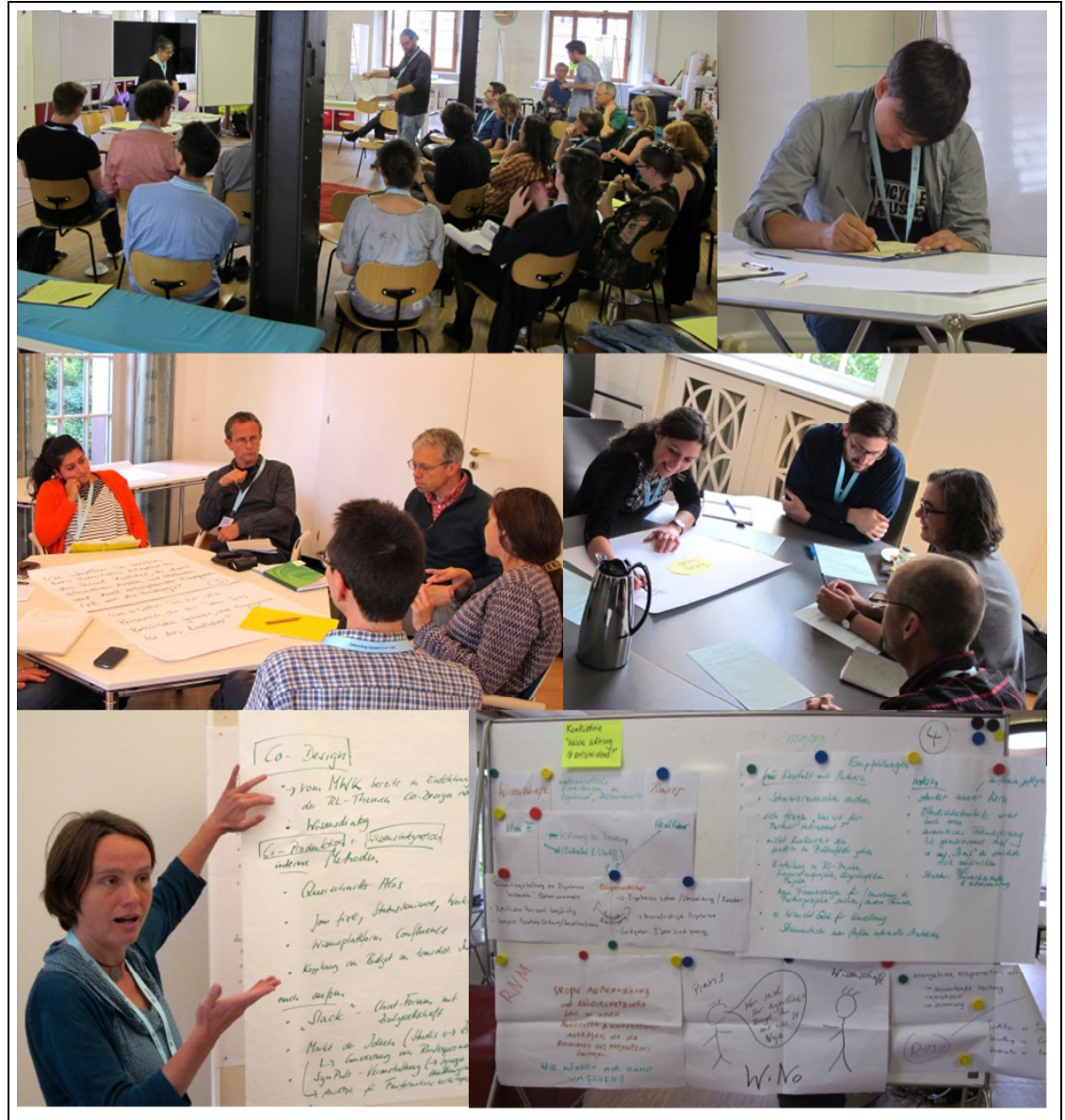

Abbildung 1: Die Treffen des Diskussionsforums waren so gestaltet, dass sich individuelle Reflexion, Reflexion innerhalb eines BaWü-Lab-Teams und BaWüLab übergreifender Austausch ergänzen. (C) BF-Team Basel

Die buchbezogenen Diskussionen waren keineswegs nur technischer Art. Obwohl es stets auch darum ging, welche Beiträge das Buch beinhalten könnte und sollte, und darum, diese Beiträge zu verbessern, führten die Fragen, die dabei aufkamen, immer auch dazu, sich im Vergleich der Erfahrungen und Argumente zu verständigen - über Partizipation, transdisziplinäres Arbeiten und transformative Forschung im Allgemeinen und über das Format Reallabor im Speziellen, über den gemeinsamen Boden und Differenzen der Bodenbeschaffenheit sowie über die Vielfalt dessen, was auf dem gemeinsamen Boden gedeiht. 
Entsprechend dürften die Fragen, die in diesen Diskussionen erörtert wurden, vermutlich über den Kreis der Personen, die sich daran abarbeiteten, hinaus von Interesse sein - und zwar unabhängig davon, ob es um Forschung im Rahmen eines Reallabors oder im Rahmen eines anderen transdisziplinären (und transformativen) Forschungsformats geht.

\section{Um was mit Blick auf die Methodik transdisziplinärer (und transformativer) Forschung gerungen wurde}

In diesem Kapitel werden drei der Fragen skizziert, die uns im Zuge der über zweijährigen Diskussionen rund um dieses Buch in besonderer Weise umtrieben. Dabei sind mit „uns“ alle gemeint, die an der Entstehung des Buchs mitwirkten, sei es als Autor(innen)en ${ }^{5}$, sei es als Mitdiskutierende an den Treffen des Diskussionsforums. ${ }^{6}$ In den Diskussionen wurden erste vorläufige Antworten auf diese Fragen gefunden - manche davon sind bereits, fertiger' als andere, bei denen sich eine Antwort erst herauszuschälen beginnt.

Die Auswahl aus den Fragen sowie die Akzente, die bei deren Erörterung gesetzt werden, sind durch die Autorin und den Autor des vorliegenden Beitrags verantwortet. Mit anderen Worten: Die Fragen als solche kamen in den persönlichen und schriftlichen Diskussionen mit den und zwischen den BaWü-Labs auf, aber die nachstehend getroffene Auswahl würde vermutlich anders ausfallen, wenn Andere als die Schreibenden die aus ihrer Sicht relevantesten Fragen zusammenstellen würden. Der Inhalt der Darstellung beruht auf dem, was in den Diskussionen mit den und zwischen den BaWü-Labs thematisiert wurde, spiegelt jedoch die Perspektiven, Eindrücke und Überlegungen der Schreibenden (mit Ausnahme der Antworten zur zweiten Frage (s. Abschnitt 4.2), die das Ergebnis der Diskussionen an einem Treffen des Diskussionsforums zusammenfassen).

Schließlich ist darauf hinzuweisen, dass für dieses Kapitel Fragen ausgewählt wurden, die mit Blick auf Aspekte der Methodik besonders bedeutsam scheinen und die gleichzeitig nicht als eigenständige Beiträge einen Niederschlag im Buch fanden (oder in anderer Weise in das Buch einflossen). Die Diskussion darüber z. B., was bei der Umsetzung des Formats Reallabor aus der Sicht derer, die Reallabore praktisch durchgeführt haben, besonders zu beachten ist, führte zu einem eigenen Beitrag im Buch (Beecroft et al. 2018), während die Frage, ob das Format Reallabor zwingend an Nachhaltigkeit als gesellschaftliches Ziel gekoppelt sein muss (die zu intensiven und durchaus kontroversen Diskussionen Anlass gab), in verschiedene Beiträge einfloss, u. a. in Kapitel 2 des vorliegenden Beitrags.

5 Diese Personen sind im Verzeichnis der Autorinnen und Autoren aufgelistet.

6 Siehe die Liste der Personen im Dank am Ende dieses Beitrags. 
Die drei Fragen, auf die nachstehend eingegangen wird, sind:

1) Wer sind die an transdisziplinärer (und transformativer) Forschung partizipierenden Akteure?

2) Wie innovativ sind die in transdisziplinärer (und transformativer) Forschung eingesetzten Methoden?

3) Können die Forschungsziele und die Praxisziele bei transdisziplinärer (und transformativer) Forschung ausbalanciert werden?

\subsection{Wer sind die an transdisziplinärer (und transformativer) Forschung partizipierenden Akteure?}

Eine Knacknuss der besonderen Art bietet die Frage, wie eigentlich die Akteure zu bezeichnen sind, die an transdisziplinärer (und transformativer) Forschung partizipieren, und welche Unterscheidungen hier sinnvoll sind.

Auf den ersten Blick einfach und einleuchtend ist, dass an solcher Forschung Personen mitwirken, die Angehörige des Wissenschaftssystems ${ }^{7}$ sind. Diese Akteure werden in der Regel als ,Forscher(innen) ' bezeichnet oder als ,Wissenschaftler(innen)'. Auf den zweiten Blick jedoch erweisen sich diese Bezeichnungen als tückisch:

- Sofern ,Forscher(innen)‘ nicht präziser gefasst wird als ,Forscher(innen), die dem Wissenschaftssystem angehören', um sie zu unterscheiden von außerwissenschaftlichen Akteuren, die sich an der Forschungstätigkeit beteiligen und so zu Mit-Forschenden werden, kann die Bezeichnung so verstanden werden, dass sie dem Grundgedanken partizipativen Forschens zuwiderläuft. Es gilt also, präzise zu sein dahingehend, welche Art ,Forscher(innen)' gemeint ist, und zu unterscheiden zwischen der Tätigkeit des Forschens und den Personen.

- ,Wissenschaftler(innen) ‘ wiederum impliziert, sofern es nicht präziser gefasst wird als ,Wissenschaftler(innen), die dem Wissenschaftssystem angehören', dass es nur innerhalb des Wissenschaftssystems Personen mit einem wissenschaftlichen Hintergrund gibt, und negiert, dass es auch außerhalb dieses Systems wissenschaftliche Expertise gibt. Stattdessen auszuweichen auf ,Ange-

7 Das Wissenschaftssystem ist nicht beschränkt auf Universitäten, Fachhochschulen u. Ä. Zum Wissenschaftssystem gehören auch außeruniversitäre Forschungseinrichtungen und -zentren wie in Deutschland z. B. das Wuppertal Institut, die Institute der Fraunhofer-Gesellschaft, der Leibniz-Gemeinschaft oder der Max-Planck-Gesellschaft, aber auch Institute wie das IÖW, das ISOE oder das Ökoinstitut. Fördergeber, die Forschung unterstützen, wie die DFG, die VolkswagenStiftung, das BMBF oder Wissenschaftsministerien auf Landesebene, gehören ebenfalls zum Wissenschaftssystem. 
hörige des Wissenschaftssystems' wäre insofern keine Lösung, als dem Wissenschaftssystem auch Personen angehören ohne einen wissenschaftlichen Hintergrund. Es gilt also, präzise zu sein dahingehend, ob sich ,Wissenschaftler(innen) ${ }^{6}$ auf den Hintergrund von Personen bezieht oder auf die kumulierten Attribute Hintergrund und Systemzugehörigkeit. Zudem gehören dem Wissenschaftssystem auch Personen an, die über einen wissenschaftlichen Hintergrund verfügen, aber nicht forschend tätig sind, was es wiederum sinnvoll oder sogar nötig macht, zusätzlich klar zu machen, ob ,Wissenschaftler(innen), die dem Wissenschaftssystem angehören', Personen meint, die forschend tätig sind, oder solche, die das nicht sind.

Solche Probleme lassen sich mit einer präzisen Begrifflichkeit durchaus vermeiden, sie scheinen also lösbar. Die Sache wird deutlich schwieriger, wenn der Blick geweitet wird auf die ,anderen' Akteure, also auf die, um die es in der partizipativen Forschung recht eigentlich geht. Das Problem fängt damit an, dass der ,Wissenschaft' ein begriffliches Gegenüber gegeben werden muss, das präziser ist als ,das Andere'. Im Angebot befinden sich hier insbesondere ,Gesellschaft', ,Zivilgesellschaft', ,außerwissenschaftlich“ und ,Praxis', die alle ihre Implikationen haben, von denen hier nur einige angesprochen werden sollen:

- Verwendet man das Begriffspaar, Gesellschaft und Wissenschaft', so impliziert dies, dass Wissenschaft nicht Teil der Gesellschaft ist, d. h. in diesem Begriffspaar bilden die beiden Teile Gesellschaft und Wissenschaft zusammen das soziale Gesamtgefüge. Wenn man sagt, bei partizipativer Forschung gehe es darum, ,gesellschaftliche Akteure' einzubeziehen, legt man mindestens implizit nahe, dass es in erster Linie darum geht, gesellschaftliche Akteure zu beteiligen, weil sie gesellschaftliche Gruppen repräsentieren, was wiederum die Vorstellung nähren könnte, partizipative Forschung habe zwingend repräsentativ zu sein (s. dazu Alcántara et al. 2018b).

- Dem Begriffspaar ,Zivilgesellschaft und Wissenschaft" wiederum liegt, sofern ,Zivilgesellschaft' als das Gegenüber von ,Staat' verstanden wird und ,Wirtschaft' beinhaltet, ein dreiteiliges Modell zugrunde, bestehend aus Zivilgesellschaft, Staat und Wissenschaft, die zusammen das soziale Gesamtgefüge bilden. Falls ,Wirtschaft' nicht als Teil der Zivilgesellschaft gesehen wird, ist das Modell vierteilig. Wenn man sagt, bei partizipativer Forschung gehe es darum, ,zivilgesellschaftliche Akteure' einzubeziehen, legt man mindestens implizit dasselbe nahe, wie wenn man sagt, es gehe darum, ,gesellschaftliche Akteure' einzubeziehen. Gleichzeitig schließt man aber bestimmte Akteurskonstellationen aus der partizipativen Forschung aus, nämlich sicher diejenigen, bei denen es Angehörige staatlicher Organe sind, die partizipieren, und je nach Begriffsverständnis auch diejenigen, bei denen wirtschaftliche Akteure partizipieren. 
- Verwendet man das Begriffspaar ,außerwissenschaftlich-wissenschaftlich', geht man vom Wissenschaftssystem als Referenzpunkt aus und definiert die Akteure, die bei partizipativer Forschung einbezogen werden, die ,außerwissenschaftlichen Akteure', ex negativo, d. h. rein dadurch, dass sie nicht Teil dieses Systems sind. Wenn man dieses Begriffspaar verwendet, kann man vermeiden, das, was sich außerhalb dieses Systems befindet, genau zu benennen. Gleichzeitig schließt man aber bestimmte Akteurskonstellationen aus der partizipativen Forschung aus, nämlich diejenigen, bei denen es Angehörige des Wissenschaftssystems sind, die partizipieren.

- Das Begriffspaar ,Praxis-Wissenschaft‘ schließlich funktioniert ähnlich wie das Begriffspaar ,außerwissenschaftlich-wissenschaftlich'. Wenn man sagt, bei partizipativer Forschung gehe es darum, ,Praxisakteure' einzubeziehen, erlaubt dies jedoch stärker als bei der Verwendung von , außerwissenschaftliche Akteure', die Beteiligung an partizipativer Forschung nicht von einer fixen Zugehörigkeit der Akteure zu einem gesellschaftlichen System abhängig zu machen, sondern von dem, was in einem Projekt erforscht wird. $\mathrm{Zu}$ sagen, dass es vom Projekt abhängt, wer Teil der ,Praxis` bzw. der ,Praxisakteure“ ist, eröffnet die Möglichkeit, dass auch Angehörige des Wissenschaftssystems partizipieren. Das Begriffspaar ,Praxis-Wissenschaft' in diesem Sinne zu verwenden, setzt allerdings voraus, dass ,Wissenschaft' nicht so sehr als System definiert wird, sondern eher als eine forschende Tätigkeit, und es setzt voraus, dass sich der Gegenstand bzw. das Thema eines Projekts in einer eingrenzbaren Praxis spiegelt. Tut man das nicht, kann das Begriffspaar ,Praxis-Wissenschaft' die Vorstellung nähren, Wissenschaft sei nicht praktisch und Praxis sei nicht wissenschaftlich.

Man muss sich also im Wissen um solche Implikationen für ein begriffliches Gegenüber der ,Wissenschaft` entscheiden, und im Bewusstsein, dass diese Entscheidung immer mit einer Gesellschaftstheorie einhergeht, und sei diese noch so grob, und mit einer Annahme darüber, wo im sozialen Gesamtgefüge sich Wissenschaft befindet (was sich selbstverständlich auch auf die Definition von ,Forscher(innen) ${ }^{`}$ und ,Wissenschaftler(innen)' auswirkt). Aufgrund dieser Theorieabhängigkeit ist in dieser Frage keine projektübergreifende Lösung möglich, lediglich Transparenz. Entsprechend wird das begriffliche Gegenüber der ,Wissenschaft‘ im vorliegenden Buch auch nicht einheitlich bezeichnet.

Auf andere Bezeichnungen wie ,Betroffene', ,Stakeholder' o. Ä. auszuweichen, ist keine wirkliche Option, weil solche Begriffe noch weniger neutral sind als die oben skizzierten und mit noch stärkeren Kriterien für die Beteiligung einhergehen: Wenn man sagt, bei partizipativer Forschung gehe es darum, dass ,Stakeholder' partizipieren oder ,Betroffene', knüpft man die Partizipation, nimmt man den Kontext, aus dem diese Begriffe stammen, ernst, entweder zwingend an Kriterien wie Mitspracherecht oder Interessen in Bezug auf das, was Anlass, Gegen- 
stand oder Ergebnis eines Projekts ist, oder zwingend an das Kriterium, auf wen sich das, was Anlass, Gegenstand oder Ergebnis eines Projekts ist, auswirkt.

Damit ist nun definitiv die Ebene eines konkreten Projekts erreicht, womit die Komplexität des Problems zunimmt. Von Unger weist darauf hin, dass sich eine bloße „Unterteilung in ,Wissenschaft‘ einerseits und (gesellschaftliche) ,Praxis“ andererseits nur bedingt [eignet]" (von Unger 2014, S. 2), um im Kontext einer im weitesten Sinne auf gesellschaftliche Wirkung ausgerichteten partizipativen Forschung sinnvoll über Partizipation reden und nachdenken zu können. Dies deshalb, weil sich die Perspektiven vervielfältigen und weil es möglich sein muss, dies zum Ausdruck zu bringen. Die drei Dimensionen, die sich in den Diskussionen rund um das vorliegende Buch für diese Vielfalt als besonders relevant erwiesen haben, sind (s. ergänzend dazu insbesondere Eckart et al. 2018, aber auch Arnold und Piontek 2018):

- Theorien gesellschaftlicher Transformation: Die Relevanz dieser Dimension zeigt sich etwa dann, wenn, in Anlehnung u. a. an das Hauptgutachten der WBGU (WBGU 2011), von „Pionieren des Wandels“ die Rede ist, die an einem Projekt partizipieren (sollen). Diese Begrifflichkeit baut letztendlich auf einer Theorie auf, wonach gesellschaftliche Transformation dadurch erfolgt, dass besonders innovative Akteure, vorausgehen' und Neues ausprobieren und so den Weg bahnen dafür, dass sich dieses Neue in der Gesellschaft ausbreiten kann. Diese Begrifflichkeit setzt, mit anderen Worten, einen diffusionstheoretischen Ansatz voraus. Ob von einer solchen oder von einer anderen Theorie gesellschaftlicher Transformation ausgegangen wird, ist projektabhängig, und die zugrunde gelegte Theorie wirkt sich natürlich darauf aus, wie über die Akteure nachgedacht und entschieden wird, die in einem Projekt partizipieren.

- Projektthema: Es ist evident, dass die Frage, welche Akteure zu unterscheiden sind, themenspezifisch zu beantworten ist, um die Partizipation in einem Projekt sinnvoll planen zu können, und dass die Differenzierungen, die dabei vorgenommen werden, zumeist nur für den Projektkontext gelten (so sind etwa filigrane Unterscheidungen verschiedener Typen von Lehrkräften nur für Projekte bedeutsam, die entsprechende Themen bearbeiten). Dies ist aber auch für Akteursbezeichnungen relevant, bei denen es möglicherweise nicht immer auf der Hand liegt: So kann es bei einem Migrations-Thema notwendig sein, differenzierte Unterscheidungen innerhalb der nicht organisierten Zivilgesellschaft vorzunehmen und dabei den Begriff Bürger(innen) nur genau für die Personen zu verwenden, die ein Bürgerrecht haben, während der Begriff Bürger(innen) bei einem anderen Thema ausreicht, um die gesamte nicht organisierte Zivilgesellschaft zu benennen. 
- Projektziele und Ziele der Partizipation: Nimmt man die Forschungsziele eines Projekts in den Blick, ist es durchaus sinnvoll, Expertise als Kriterium der Beteiligung anzulegen und die an einem Projekt partizipierenden Akteure entsprechend auszuwählen (s. dazu Defila und Di Giulio 2018c). Nimmt man hingegen die Praxisziele in den Blick, greift das Kriterium der Expertise zu kurz, weil Expertise nicht (allein) ausschlaggebend sein dürfte, wenn es z. B. darum geht, die gesellschaftspolitische Legitimität transformativer Aktivitäten oder die praktische Relevanz von Ergebnissen und Produkten sicherzustellen (s. dazu auch Defila und Di Giulio 2018a), oder wenn es darum geht, partizipativ aus zivilgesellschaftlichen Initiativen Ideen für Realexperimente zu generieren. Welche Akteure zu unterscheiden sind und welche Kriterien der Beteiligung anzuwenden sind, ist also zum einen abhängig von den Zielen auf der Ebene eines Projekts, wobei mindestens zwischen Forschungs- und Praxiszielen zu differenzieren ist. Zum anderen ist es abhängig von den Zielen der Partizipation, die bezogen auf die einzelnen Aktivitäten in einem Projekt verfolgt werden.

Um die Vielfalt der Perspektiven, die in ein transdisziplinäres (und transformatives) Forschungsprojekt einbezogen werden sollen, angemessen zum Ausdruck zu bringen, ist eine auf das konkrete Projekt bezogene Typologie von Akteuren unerlässlich. Diese muss auf die zugrunde gelegte Transformationstheorie, das Projektthema, die Projektziele sowie die konkreten Partizipationsziele der verschiedenen Projektaktivitäten zugeschnitten sein. Schließlich und endlich wird diese Typologie auch die in einem Projekt zugrunde gelegten Partizipationstheorien spiegeln (Alcántara et al. 2018b). Das ist für ein individuelles Projekt erforderlich und auch leistbar.

$\mathrm{Ob}$ es hingegen leistbar ist, eine Typologie von Akteuren vorzulegen, die sowohl einen generellen Anspruch erheben kann als auch überzeugend differenziert ist, bleibt zu prüfen. Angesichts der Vielfalt und Differenziertheit der realen Partizipation in den BaWü-Labs scheint es zumindest fraglich, ob mehr produziert werden könnte als allgemein-abstrakte und immer auch unabgeschlossene Listen von Akteurs-Typen. Entsprechend finden sich im vorliegenden Buch durchaus verschiedene Akteurs-Typologien.

Die Beschäftigung mit der Knacknuss, wie eigentlich die Akteure zu bezeichnen sind, die an transdisziplinärer (und transformativer) Forschung partizipieren, und welche Unterscheidungen hier sinnvoll sind, führte also zum Ergebnis, dass dafür (zumindest: noch) keine allgemein verwendbaren und gleichzeitig angemessen differenzierten Vorschläge vorgelegt werden können - wohl aber Überlegungen und Fragen, die bei der Planung und Gestaltung der Partizipation in einem solchen Projekt dienlich sein können (s. Beecroft et al. 2018 und Eckart et al. 2018). Sie führte aber auch, zumindest in der Wahrnehmung der Schreibenden, zu einer 
Sensibilisierung für die Stolpersteine, die mit dem Reden über diese Frage verbunden sind, und zur Einsicht, dass die kritische Durchleuchtung der dabei verwendeten Begriffe keine Haarspalterei darstellt, sondern handlungsrelevant ist, weil es nicht nur unmittelbar damit zusammenhängt, wer partizipiert, sondern auch damit, welchen Zwecken Partizipation dienen soll und kann.

Schließlich wurde aber auch klar, dass es gilt, in der Auseinandersetzung mit und in der Kommunikation über diese Frage drei Dinge zu vermeiden: Zu komplexe Modelle des sozialen Gefüges zu entwerfen, im Versuch, die Komplexität zu reduzieren, bei zu simplen Bildern des sozialen Gefüges zu landen, im Vermeiden von Beidem zu verstummen.

Die Partizipation in einem Projekt muss zu guter Letzt ihren Niederschlag in der Projektstruktur finden. Entsprechend bot die Frage der Projektorganisation Anlass zu Diskussionen zwischen den und mit den BaWü-Labs. Ist die Vielfalt der Akteure und Kooperationsformen so groß und dynamisch, wie dies bei den BaWüLabs der Fall war bzw. ist (und wie es vermutlich in Reallaboren generell der Fall ist), ist unmittelbar einleuchtend, dass es einer entsprechend komplexen Projektstruktur bedarf. Dabei ist auch in Rechnung zu stellen, dass sich die Intensität und Dauer der Mitwirkung in einem partizipativen Forschungsprojekt von Akteur zu Akteur unterscheiden kann und zumeist auch muss (s. für transdisziplinäre Forschung bereits Defila et al. 2006, S. 216f.). Sich mit diesen unterschiedlichen Intensitäten der Zusammenarbeit und mit den damit einhergehenden Unterschieden hinsichtlich der Nähe zum Projektgeschehen zu befassen, ist unerlässlich, schon allein, um nicht dem Irrtum anheim zu fallen, zu meinen, Partizipation bedeute, dass alle alles machen müssten (Seebacher et al. 2018b) oder dass immer alle über alles informiert sein müssten (Quint et al. 2018). Ein Aspekt, der sich bei einigen der BaWü-Labs ebenfalls als wichtig erwies und der daraus entsteht, dass die transdisziplinäre und transformative Arbeit von Reallaboren sehr unmittelbar ,in die reale Welt' eingebettet ist, betrifft das Management der ,Außengrenzen' zu eben dieser ,realen Welt'. Die Zusammenführung all dieser Aspekte, d. h. der Vielfalt der Akteure, der Diversität und Dynamik der Kooperationsformen und -intensitäten sowie der Bedeutung der ,Außengrenzen', mündete in den Vorschlag eines Modells der Akteurskonstellation in einem Reallabor (s. Seebacher et al. 2018a). Ein Modell, das dienlich sein kann dafür, wie an die Gestaltung der Projektstruktur eines Reallabors herangegangen werden könnte - und das wohl auch für andere transdisziplinäre (und transformative) Kontexte eine Denkhilfe darstellen dürfte. 


\subsection{Wie innovativ sind die in transdisziplinärer (und transformativer) Forschung eingesetzten Methoden?}

Wird Forschung transdisziplinär, also partizipativ und interdisziplinär, betrieben, kommen nicht nur die Methoden der Wissensgenerierung zum Einsatz, die zum traditionellen Kanon disziplinärer Forschung gehören, sondern auch Methoden, die in vielen disziplinären Kontexten (noch) ungewohnt sind, bzw. Methoden, die es neu zu erfinden bzw. neu zu kombinieren gilt (s. dazu Defila und Di Giulio 2018c). Solche Methoden sind, grob gesagt, zumeist darauf ausgerichtet, verschiedene Wissensbestände sichtbar zu machen und aufeinander zu beziehen, den Akt der Kommunikation in mehr oder weniger großen und heterogenen Gruppen von Menschen durch Reflexion und Dialog mit Blick auf forschungsrelevante Entscheidungen zu strukturieren sowie Wissen zu erzeugen durch die Integration vielfältiger Wissensbestände und Perspektiven. Verfolgt ein Forschungsvorhaben nicht nur Ziele der Erkenntnisgewinnung, sondern auch transformative Ziele, spielen bei der Wahl und Umsetzung von Methoden zusätzliche Aspekte wie das Anstoßen, Konzipieren und Umsetzen transformativer Aktivitäten (und damit auch die Ansprache und Aktivierung der dafür relevanten Akteure) eine Rolle. Entsprechende Methoden sind naturgemäß oft ,sichtbarer' als etwa die Befragung mittels Fragebögen oder Interviews oder die Analyse von Publikationen und Dokumenten, und zwar insbesondere dann, wenn sie mit dem Einbezug einer größeren Gruppe von Menschen einhergehen. Dies gilt umso mehr, wenn solche Methoden, wie dies bei einer transformativen Forschung in der Regel der Fall ist, einhergehen oder zusammenfallen mit Interventionen ,in der realen Welt', die nicht nur zur Generierung wissenschaftlichen Wissens dienen sollen, sondern auch dazu, Transformationsprozesse in der Gesellschaft anzustoßen. Entsprechende Vorgehensweisen ziehen aber nicht nur mehr Aufmerksamkeit auf sich (und in der Wissenschaft eine gewisse Skepsis, was den Stellenwert des dabei erzeugten Wissens betrifft), sie haben in vielen Kontexten auch Neuigkeitswert und/oder Unterhaltungswert.

Das mag erklären, weshalb solche Methoden oft als innovativ bezeichnet werden. Im ersten Treffen des Diskussionsforums im Oktober 2015 wurde diskutiert, ob bzw. inwieweit dies gerechtfertigt ist. ${ }^{8}$ Diese Diskussion ergab, dass hier in dreierlei Hinsicht Zurückhaltung geboten ist:

- Erstens ist ,innovativ' relational und daher hier auf einer sehr grundsätzlichen Ebene potentiell irreführend. $\mathrm{Ob}$ eine Methode ,innovativ' ist, bemisst sich

8 Was nachstehend (bis zum Ende von Abschnitt 4.2) dargestellt wird, ist eine Zusammenfassung der Ergebnisse dieses Treffens, festgehalten im internen Dokument „Innovative Methoden und Methodenwahl. Vorgehen und Auswertung der Diskussion“ vom 03.02.2016, das von der BF-Basel erstellt wurde. 
meist nicht an der Methode selbst, sondern daran, ob sie in einem bestimmten Kontext bzw. in einer bestimmten Gruppe bereits bekannt ist und verwendet wird. Methoden können also immer nur bezogen auf einen bestimmten Kontext als innovativ bezeichnet werden, und dies setzt gleichzeitig die Existenz einer zumindest halbwegs homogenen Gruppe voraus. Angesichts der Tatsache, dass ein transdisziplinäres (und transformatives) Vorgehen nicht auf eine homogene Gruppe von Wissenschaftler(inne)n beschränkt ist, ist es streng genommen nicht möglich (und bringt keinen ersichtlichen Mehrwert), kontextfrei von einer innovativen Methode zu sprechen. Es könnte im Gegenteil sogar als anmaßend wahrgenommen werden und Ablehnung hervorrufen.

- Zweitens könnte das diskursive Zusammenschließen von transdisziplinärer (und transformativer) Forschung auf der einen Seite und der Innovativität von Methoden auf der anderen Seite dazu führen, dass partizipative Forschung, und insbesondere solche, die auch transformativ sein will, gleichgesetzt wird mit dem Einsatz ,neuer' (und möglichst spektakulärer) Methoden. Das wiederum könnte mit sich bringen, dass die Qualität solcher Forschung gleichgesetzt wird mit dem Maß an Spektakel und/oder dass bezogen auf diese Forschung ein Zwang zum Spektakulären entsteht. Beides könnte die Vorstellung nähren, dass hier die Neuheit von Methoden höher gewichtet werde als deren Eignung und Passung mit Blick auf die Ziele, die ein Projekt anstrebt, und mit Blick auf die Fragen, die forschend und experimentierend beantwortet werden sollen. Das wiederum wäre nicht sachgerecht und würde überdies den Vorbehalten Vorschub leisten, die innerhalb der Wissenschaft gegenüber solcher Forschung bestehen.

- Drittens könnte die Fokussierung auf ,neue' Methoden den Blick darauf verstellen, dass sich partizipative Forschung, unabhängig davon, ob sie auch transformativ ist oder nicht, durchaus des Kanons traditioneller Methoden bedient. Es werden sogar größtenteils bekannte und bewährte Methoden verwendet. Das ,Neuartige' und für Viele Ungewohnte ergibt sich oftmals weniger daraus, dass Methoden in einem Kontext zum Einsatz gelangen, in dem diese vielleicht unüblich bzw. unbekannt sind, sondern eher aus der Art und Weise, wie diese Methoden kombiniert werden, aus deren situativer Anpassung oder aus der Abfolge, in der sie eingesetzt werden.

Dienlicher als eine Debatte über die Neuartigkeit der Methoden, die bei transdisziplinärer (und transformativer) Forschung eingesetzt werden, scheint daher eine Auseinandersetzung mit Fragen wie der, wofür sich welche Methode unter welchen Bedingungen eignet, wie sichergestellt werden kann, dass bei der Umsetzung von Methoden die erforderliche Qualität der Prozesse und Ergebnisse erreicht wird, und wie Vorbehalten gegenüber den erzielten Ergebnissen in der Wissenschaft begegnet werden könnte. Methoden nehmen, das wurde auch von den BaWü-Labs, die am Treffen dabei waren, betont, eine Schlüsselbedeutung 
ein mit Blick auf die innerwissenschaftliche Akzeptanz dessen, was in transdisziplinären Forschungsprojekten sowie in transdisziplinären und transformativen Forschungsformaten wie dem Format Reallabor getan wird.

Die sorgfältige Reflexion des methodischen Vorgehens wiederum, die auf Nachvollziehbarkeit ausgerichtete Darstellung des methodischen Vorgehens und die kritische Methodendiskussion werden, so die Einschätzung am Treffen des Diskussionsforums, im Arbeitsalltag jedoch oft vernachlässigt. Dies ist insbesondere dann der Fall, wenn nicht nach ,Schema $X^{`}$ vorgegangen werden kann, sondern situativ und in Echtzeit über das weitere Vorgehen entschieden werden muss, z. B. im Rahmen einer Intervention ,in der realen Welt (bei Reallaboren in der Form von Realexperimenten). Methodische Entscheidungen werden in solchen Situationen zwar nicht unüberlegt, oft aber intuitiv getroffen, und die Forschenden sind Teil des Prozesses, den sie gestalten. Entsprechend bedeutsam sind die (nachträgliche) Reflexion und Dokumentation von Entscheidungen, und zwar nicht nur mit Blick auf die wissenschaftliche Nachvollziehbarkeit, sondern auch mit Blick auf die für die wissenschaftliche Arbeit erforderliche Distanz zum Geschehen. Mündet eine solche rekonstruktive Auseinandersetzung in die Aufbereitung von Methoden, stellt dies über das einzelne Forschungsprojekt hinaus einen Beitrag zur wissenschaftlichen Güte solcher Forschung dar. Die Arbeit am vorliegenden Buch wurde als Gelegenheit gesehen, über die Wahl und Umsetzung von Methoden im eigenen BaWü-Lab zu reflektieren und gleichzeitig einen methodologischen Beitrag über das eigene BaWü-Lab hinaus zu leisten.

\subsection{Können die Forschungsziele und die Praxisziele bei transdisziplinärer (und transformativer) Forschung ausbalanciert werden?}

Reallabor-Projekte sind inter- und transdisziplinär, und sie verfolgen, wie bereits verschiedentlich erwähnt, Ziele in mindestens zwei, im Idealfall drei Dimensionen: Forschungsziele, also die Erzeugung neuen Wissens, Praxisziele, also das Anstoßen und Begleiten von Transformationsprozessen, und Bildungsziele, also das Anregen und Unterstützen von Lernprozessen (s. Beecroft et al. 2018). Entsprechend haben es Reallabore mit kumulativen Herausforderungen zu tun: Erstens die Herausforderung, interdisziplinär zu sein. Zweitens die Herausforderung, transdisziplinär zu sein. Drittens die Herausforderung, transformativ und forschend gleichzeitig zu sein. Eine solche Vielfalt von Zielen in der konkreten Projektrealität auszubalancieren und dabei mit diesen kumulierten Herausforderungen umzugehen, ist nicht einfach, weder mit Blick auf das Design eines Projektes noch mit Blick auf die Ressourcenallokation noch mit Blick auf die in einem Projekt verwendeten Methoden, die im Idealfall der Erreichung von Zielen in allen drei Dimensionen dienen. 
Die Herausforderungen, die mit inter- und transdisziplinärem Arbeiten einhergehen, sind sattsam bekannt und müssen hier nicht ausgebreitet werden, ebenso wenig wie die Herausforderungen, die generell mit partizipativer Forschung einhergehen. Letzteres betrifft auch die Diskussion darüber, inwieweit Wissen, das aus Einzelfällen gewonnen wird, generalisierbar ist - auch das wird in der Literatur sowohl zu partizipativer Forschung als auch zu Reallaborforschung breit diskutiert und muss hier nicht wiederholt werden. Die Diskussion mit den und zwischen den BaWü-Labs haben diese Herausforderungen bestätigt.

Was an dieser Stelle betont werden soll, ist ein Aspekt, der sich auch in vielen der Vorgehensweisen spiegelt, die in BaWü-Labs umgesetzt wurden (bzw. werden): Realexperimente (oder analoge Aktivitäten), die konzipiert und umgesetzt werden mit Blick auf die Transformationsprozesse, die angeregt, begleitet und erforscht werden sollen, spielen in einem transformativen Forschungsprojekt eine zentrale Rolle. Solche Realexperimente werden in Reallaboren aber zumeist nicht allein aus der Forschungslogik und aus Forschungsbedarfen heraus konzipiert, sondern stark aus der Logik und den Bedarfen der ,realen Welt' heraus, in die sie eingebettet sind. Werden solche Realexperimente (oder analoge Aktivitäten) nach dieser doppelten, aber klar gewichteten Logik generiert, kann jedoch nicht davon ausgegangen werden, dass sie sich leicht in Forschungsbedarfe einfügen, d. h. dass sie in Ausrichtung und Konzeption erlauben, Fragen zu erforschen, die zum wissenschaftlichen Fortschritt beitragen. Vielmehr bedarf es einer besonderen Anstrengung, diese mit Forschungsbedarfen zu ,versöhnen'. Wie leicht oder schwer dies gelingt, hängt davon ab, wie in einem Projekt bei der Entwicklung von Realexperimenten (oder analogen Aktivitäten) diese beiden Logiken im Verhältnis zueinander gewichtet werden. Und manchmal dürfte es auch nicht gelingen, d. h. manchmal werden Bedarfe nach wissenschaftlich generierten Informationen oder partizipativ entwickelte transformative Aktivitäten nicht in Übereinstimmung gebracht werden können mit Fragen, deren Antworten zum wissenschaftlichen Fortschritt beitragen.

Entsprechend ist das Ringen um Methoden, die sich für eine transdisziplinäre (und transformative) Forschung eignen, immer auch ein Ringen um die Balance zwischen Forschungszielen, Praxiszielen und Bildungszielen - und das spiegelt sich auch in den Vorgehensweisen, die von BaWü-Labs für Teil 2 dieses Buchs aufbereitet wurden.

\section{Zum Aufbau des Buchs}

Ziel des vorliegenden Buchs ist es, wie eingangs erwähnt, einen Beitrag zu leisten zur Methodik transdisziplinärer (und transformativer) Forschung. Dies gliedert sich in zwei Teile, auf die nachstehend kurz eingegangen wird (s. auch Liste am Ende dieses Beitrags). 


\section{Teil 1}

Im ersten Teil des Buchs werden Kriterien, Fragen und Prinzipien mit Blick auf die Planung und Gestaltung transdisziplinärer (und transformativer) Forschung vorgestellt. Diese gründen in den Erfahrungen der BaWü-Labs und entstanden als Ergebnis des Austauschs zwischen BaWü-Labs, den gemeinsamen Reflexionen und dem Ringen um Gemeinsamkeiten und Unterschiede. Diese Beiträge wurden, soweit es sich um solche von BaWü-Labs handelt, durch gemischte Teams aus mindestens zwei BaWü-Labs verfasst. Die Reihenfolge, in der im Folgenden die Beiträge vorgestellt werden, entspricht nicht der Reihenfolge, wie sie im Buch abgedruckt sind.

Drei Beiträge fokussieren auf das Format Reallabor:

- Der Beitrag Zentrale Begriffe im Kontext der Reallaborforschung von Annika Arnold und Felix M. Piontek erläutert Begriffe, die sich im Reallabordiskurs als besonders wichtig herausgeschält haben. Dabei wird auf Begriffe fokussiert, die für viele Beiträge in diesem Buch eine wichtige Rolle spielen und bei denen gleichzeitig entschieden wurde, sie nicht in jedem dieser Beiträge wieder im Detail auszubreiten.

- Der Beitrag Reallabore als Rahmen transformativer und transdisziplinärer Forschung: Ziele und Designprinzipien von Richard Beecroft, Helena Trenks, Regina Rhodius, Christine Benighaus und Oliver Parodi befasst sich damit, was Reallabore kennzeichnet mit Blick auf deren Ziele, und schlägt Prinzipien vor, die bei der Konzipierung, beim Aufbau und beim ,Betrieb“ eines Reallabors berücksichtigt werden sollten. Dabei wird nicht aus der Theorie argumentiert, sondern aus der reallaborpraktischen Erfahrung.

- Der Kurz-Beitrag Akteure in Reallaboren - Reallabore als Akteure von Andreas Seebacher, Sophia Alcántara und Alexandra Quint schlägt ein Modell vor, anhand dessen die Akteurskonstellation in einem Reallabor mit Blick auf die Projektstruktur analysiert werden kann. Das Modell berücksichtigt die Dynamik und Fluktuation, die in einer solchen Akteurskonstellation nicht die Ausnahme, sondern die Regel ist.

Vier Beiträge fokussieren auf Partizipation:

- Der Beitrag Leitfragen für die Gestaltung von Partizipationsprozessen in Reallaboren von Jochen Eckart, Astrid Ley, Elke Häußler und Thorsten Erl schlägt Fragen für die Planung von Partizipationsprozessen vor. Diese decken die Aspekte Erfolgsfaktoren, Beteiligungsziele, Akteursanalyse und Ablauf von Partizipationsprozessen ab und wurden gewonnen aus dem Zusammenführen von Ansätzen aus der gesellschaftspolitisch-planerischen Partizipation und Reallabor-Erfahrungen. 
- Der Kurz-Beitrag Der Partizipationsmythos „,Partizipation in Reallaboren muss repräsentativ sein "von Sophia Alcántara, Alexandra Quint und Andreas Seebacher erörtert den Mythos, Partizipation in der Forschung müsse immer repräsentativ sein und Repräsentativität bedeute dabei immer das (statistische) Abbilden der Gesellschaft. Zudem zeigt er auf, wie Vorstellungen über Partizipation mit demokratietheoretischen Ansätzen zusammenhängen.

- Der Kurz-Beitrag Der Partizipationsmythos „Partizipation in Reallaboren ist per se transparent und muss es auch sein " von Alexandra Quint, Sophia Alcántara und Andreas Seebacher erörtert den Mythos, Partizipation in der Forschung müsse, analog zur gesellschaftspolitisch-planerischen Partizipation, immer transparent sein, so dass immer alle über alles informiert sind. Er zeigt, dass dies nicht nur unmöglich wäre, sondern manchmal sogar die Zusammenarbeit behindern würde.

- Der Kurz-Beitrag Der Partizipationsmythos „Partizipation bedeutet, alle immer an allem zu beteiligen " von Andreas Seebacher, Sophia Alcántara und Alexandra Quint erörtert den Mythos, Partizipation in der Forschung sei nur dann gut, wenn alle an möglichst allen Aktivitäten mitwirken würden und an allen Entscheidungen beteiligt seien. Er zeigt, dass das nicht nur alle Beteiligten überfordern, sondern auch nicht den tatsächlichen Partizipationsbedarfen entsprechen würde.

Ein Beitrag fokussiert auf die Methoden der partizipativen Wissenserzeugung:

- Der Beitrag Partizipative Wissenserzeugung und Wissenschaftlichkeit - ein methodologischer Beitrag von Rico Defila und Antonietta Di Giulio schlägt, auf der Grundlage einer Literaturanalyse, einen Kanon von Gütekriterien für Methoden der partizipativen Wissenserzeugung in der transdisziplinären Forschung vor. Drei Kriterien beziehen sich auf das Ziel und den Kontext von Methoden, acht auf die Umsetzung von Methoden, zwei auf die Reflexion der Erfahrungen.

\section{Teil 2}

Im zweiten Teil des Buchs werden ausgewählte Methoden, die in BaWü-Labs entwickelt und umgesetzt wurden und die sich besonders bewährt haben, für die transdisziplinäre (und transformative) Forschung innerhalb und außerhalb von Reallaboren zur Verfügung gestellt. Dabei wurde besonders Wert gelegt auf Methoden, die nicht allein Forschungszielen oder Praxiszielen oder Bildungszielen dienen, sondern Zielen in mehr als einer dieser Dimensionen. Diese Methoden werden ausführlich beschrieben, und zwar so, dass Dritte sie umsetzen können. 
Dabei wurden die Gütekriterien zugrunde gelegt, die im Beitrag von Defila und Giulio im ersten Teil des Buchs entfaltet werden. Zudem wurde darauf geachtet, die Vorgehensweisen so aufzubereiten, dass sie in vielfältigen Kontexten umsetzbar sind. Die Beiträge sind keine Fallstudien, d. h. die Erfahrungen aus dem jeweiligen BaWü-Lab dienen, sofern solche dargestellt werden, primär dem Zweck der vertiefenden Illustration. Die Reihenfolge, in der im Folgenden die Beiträge vorgestellt werden, entspricht der Reihenfolge, wie sie im Buch abgedruckt sind.

Per „Wissensmesse“ zum Forschungsprogramm - Arbeitsphasen und Abstimmungsprozesse zwischen Wissenschaft und Praxis von Regina Rhodius und Michael Pregernig

Die „Wissensmesse“ führt in mehreren Schritten in einem kompetitiven Verfahren zu Forschungsfragen, die sowohl Bedarfe von Praxisakteuren als auch Forschungsbedarfe adressieren. Praxisakteure sind hier organisierte Öffentlichkeit und Fachöffentlichkeit (nicht die breite Öffentlichkeit). Das Vorgehen ist zuerst interdisziplinär, dann transdisziplinär, d. h. der Kreis der Beteiligten wird sukzessive erweitert. Ein Projektbeirat unterstützt den Auswahlprozess.

Der Weg zum Realexperiment - Schlüsselakteure identifizieren, Kooperationsstrukturen aufbauen, Projektideen auswählen von Eric Puttrowait, Raphael Dietz, Marius Gantert und Johannes Heynold

Das Vorgehen ist in allen seinen Schritten partizipativ angelegt und erlaubt den Einbezug von Studierenden. Es dient dazu, Ideen zivilgesellschaftlicher Akteure für Realexperimente aufzugreifen und transdisziplinär weiterzuentwickeln $\mathrm{zu}$ Vorschlägen, aus denen dann diejenigen ausgewählt werden, die als Realexperimente umgesetzt werden (sollen). Dies erfolgt in einem mehrteiligen kompetitiven Verfahren, und für die Auswahl der Realexperimente wird eine unabhängige Jury eingesetzt.

Mit einer Realexperimentreihe Impulse für soziale Innovationen setzen - Realexperimente initiieren, begleiten und beforschen von Helena Trenks, Colette Waitz, Sarah Meyer-Soylu und Oliver Parodi

Eine Realexperimentreihe besteht aus mehreren gleichzeitig laufenden Realexperimenten, die allesamt auf Ideen und Initiativen zivilgesellschaftlicher Akteure zurückgehen. Das Auswahlverfahren ist kompetitiv, die Ideen werden mittels eines offenen Wettbewerbs eingeholt. Die transdisziplinäre (und transformative) Arbeit intensiviert sich nach der Auswahl der Realexperimente, wenn die Akteure, die diese umsetzen, auf ihrem Weg begleitet werden und die Realexperimente erforscht werden. 
Zwischen Wunsch und Wirkung - Ein transdisziplinärer Visionsworkshop mit Bürgerinnen und Bürgern von Sophia Alcántara, Annika Arnold, Doris Lindner, Sigrid Busch, Raphael Dietz, Markus Friedrich, Charlotte Ritz und Marco Sonnberger

Eine Reihe ineinandergreifender partizipativer und interdisziplinärer Schritte führt zu Vorstellungen einer wünschenswerten Zukunft, in die sowohl qualitative Visionen wie auch deren modellierte und visualisierte Auswirkungen einfließen. Durch die Modellierung werden die Visionen von Bürger(inne)n in quantitative Größen übersetzt, und die Visualisierung macht deren Auswirkungen sichtbar. Dies bildet die Grundlage für eine transdisziplinäre Bewertung.

Die ,WiNo-Methode“ zur Identifikation von Wissen in transdisziplinären Netzwerken mithilfe der Wissensbilanzierung von Monika Bachinger, Daniel Bleher, Hannes Rau und Rasmus Prieß

Die „WiNo-Methode“ dient dazu, das Wissen in typischerweise unverfassten transdisziplinären Netzwerken zu erfassen. Dieses Wissen wird gegliedert nach Humankapital (das Wissen der Akteure zum Thema, das erforscht werden soll), Strukturkapital (Ressourcen wie z. B. Treffpunkte oder Netzwerkwissen, die das Netzwerk der Akteure als Gesamtheit aufweist) und Beziehungskapital (Wissen, Erfahrungen, Lernimpulse etc., die das Netzwerk von außen erhält).

„Wissen to Go“ - Transdisziplinär-transformative Lehre als „Reallabor im Kleinen" von Christina West

„Wissen to Go“ zeigt, wie der Reallabor-Ansatz konsequent in eine Lehrveranstaltung übersetzt werden kann. Thematischer Rahmen sind Wissensproduktion bzw. Wissen. Die Studierenden erarbeiten zuerst in einem interdisziplinären Schritt Wissen zu dieser Thematik und erste Ideen für Realexperimente. Anschließend suchen sie die transdisziplinäre Zusammenarbeit mit Praxisakteuren, entwickeln mit diesen ihre Ideen für Realexperimente weiter, setzen diese um und beforschen sie.

\section{Was sich aus Erfahrungen in Reallaboren lernen lässt für transdisziplinäre (und transformative) Forschung}

Das Format Reallabor hat das Potential, Impulse zu setzen für eine neue Diskussion der alten Frage nach der gesellschaftlichen Rolle von Wissenschaft und Forschung und so Veränderungen (auch) im Wissenschaftssystem anzustoßen. Es hat aber auch das Potential, Impulse zu setzen für das Anliegen, das hier im Vordergrund steht, nämlich zur Methodik transdisziplinärer (und transformativer) Forschung beizutragen. 
Abschließend soll dargelegt werden, welches aus Sicht der Autorin und des Autors wesentliche Beiträge von Reallaboren zur Methodik transdisziplinärer (und transformativer) Forschung sind. Diese Zusammenstellung spiegelt den Reflexionsstand der Schreibenden und beansprucht nicht, mehr zu sein als eine Anregung für die künftige Diskussion. Genährt ist sie aus Diskussionen mit Kolleg(inn)en in den BaWü-Labs und im zweiten BF-Team, aus Ergebnissen der Diskussionen zwischen den BaWü-Labs und aus der gemeinsamen Arbeit am vorliegenden Buch mit den Kolleg(inn)en aus der Mitte der BaWü-Labs:

Reallabore lenken den Blick auf die Notwendigkeit, Partizipation und Kooperation sehr feinmaschig zu denken. Die Akteurskonstellation ist in Reallaboren komplex, differenziert und dynamisch. Dies betrifft die Partizipationsziele ebenso wie die Akteurs-Vielfalt, die Vielfalt der Kooperationsformen ebenso wie die Rollen, die die Beteiligten haben, und deren Legitimation. Um dies zu erfassen, bedarf es einer differenzierten Herangehensweise an die Bezeichnung der partizipierenden Akteure und an die Bestimmung von Kriterien der Beteiligung, die erlauben, eine Vielfalt von Partizipationszielen abzudecken. Um damit umzugehen, bedarf es differenzierter und elaborierter Vorgehensweisen, beides umfassend, Heuristiken und Methoden.

Reallabore können Methoden zur Verfügung stellen, die sich in besonderer Weise dazu eignen, gleichzeitig sowohl erkenntnisbezogene Ziele als auch transformationsbezogene Ziele zu erreichen. Die doppelte, wenn nicht dreifache, Zielsetzung von Reallaboren macht es notwendig, dass in entsprechenden Projekten ein reflektierter Umgang mit Methoden erfolgt dahingehend, dass für jede Vorgehensweise auszutarieren ist, wie diese Ziele zu gewichten sind und wie vorzugehen ist, um diesen Zielen gleichzeitig Rechnung zu tragen. Da die Aktivitäten von Reallaboren stark in die ,reale Welt' eingebettet sind, darf erwartet werden, dass die in Reallaboren gewählten Vorgehensweisen in besonderer Weise zur partizipativen Qualität von Methoden der Wissensproduktion beitragen.

Reallabore könnten einen institutionalisierten Ort bieten, um sich mit der Methodik transdisziplinärer (und transformativer) Forschung zu befassen. Reallabore werden im Diskurs verschiedentlich als Infrastruktur für transdisziplinäre (und transformative) Projekte verstanden. Würden Reallabore entsprechend konzipiert und auf längere Zeit verstetigt, könnten sie damit zu Einrichtungen werden, in denen eine systematische Auseinandersetzung mit methodologischen Fragen stattfinden kann und in denen Methoden für die transdisziplinäre Forschung entwickelt, getestet und aufbereitet werden können, idealerweise sowohl für eine transdisziplinäre Forschung, die transformativ sein will, als auch für eine, die das nicht (oder mindestens nicht im selben Maß) sein will. 
Reallabore sind eine reiche Quelle, aus der mit Blick auf die Methodik transdisziplinären (und transformativen) Forschens viel geschöpft werden kann. Das Forschungsformat hat, trotz oder gerade wegen einer gewissen Sperrigkeit, das Potential, weitere Impulse auch und gerade für Wissenschaft und Forschung zu setzen - sofern diese bereit sind, aus den Erfahrungen in den und mit den Reallaboren zu lernen.

\section{Dank}

Dieses Buch trägt den Stempel derer, die aus der Mitte der BaWü-Labs über Sinn und Zweck des Buchs, über dessen Inhalt und Aufbau diskutierten, unabhängig davon, ob sie am Ende selbst als Autor(inn)en tätig wurden. Die offenen und vertrauensvollen, inspirierenden und durchaus kontroversen Diskussionen lieferten den Nährboden, auf dem das wachsen konnte, was sich nun zwischen zwei Buchdeckeln findet. Dies sind:

Marius Albiez, Sophia Alcántara, Annika Arnold, Monika Bachinger, Thomas Becker, Carolin Becker-Leifhold, Richard Beecroft, Michael Bossert, Raphael Dietz, Jochen Eckart, Thorsten Erl, Marius Gantert, Ulrike Gerhard, Maximilian Haag, Anja-Lisa Hirscher, Constantin Hörburger, Michael Kalff, Barbara Koch, Jan A. Lutz, Editha Marquardt, Martin Müller, Aida Nejad, Hanna Noller, Oliver Parodi, Felix M. Piontek, Michael Pregernig, Eric Puttrowait, Regina Rhodius, Clemens Rudolf, Ines-Ulrike Rudolph, Holger Schallehn, Andreas Schmitt, Friedemann Schwenkreis, Andreas Seebacher, Antje Stokman, Helena Trenks, Colette Waitz, Christina West, Stefan Zimmermann.

Ein großer Dank geht an den Reigen der Personen, die sich im Rahmen des externen Reviews vertieft mit dem Ziel des Buchs und den einzelnen Beiträgen befassten. Deren Einlassung auf die Idee des Buchs und deren wohlwollend-kritische Prüfung trugen wesentlich zur Qualität des Buchs bei. Um die Anonymität des Reviews zu wahren, kann den Gutachter(inne)n bei den einzelnen Beiträgen nicht namentlich, sondern lediglich abstrakt gedankt werden. Namentlich genannt sind sie aber im Verzeichnis der Review-Personen.

Danken möchten wir auch Anna-Lena Hermelingmeier und Angelika Schulz für Korrektorat und Satz und vor allem für die Geduld, mit der sie die dynamische Produktion des Buches ertragen haben.

Ein besonderer Dank geht schließlich an das Ministerium für Wissenschaft, Forschung und Kunst Baden-Württemberg (MWK), ohne dessen Förderung weder die Arbeit in den BaWü-Labs noch die Arbeit der BF-Basel möglich gewesen wären und ohne dessen Unterstützung auch dieses Buch nicht entstanden wäre. 


\section{Literatur}

Bäckstrand, K. (2003). Civic Science for Sustainability: Reframing the Role of Experts, Policy-Makers and Citizens in Environmental Governance. Global Environmental Politics, 3 (4), (S. 24-41).

BGL-Themenheft (2017). Reallabore als Forschungsformat nachhaltiger Stadtentwicklung. Berichte. Geographie und Landeskunde, 91 (1). (im Druck).

Burton, R., Ronningen, K., \& Wedderburn, L. (2008). Conducting integrated research. A critical literature review of interdisciplinary and transdisciplinary research. Report 12/08. Trondheim: Centre for Rural Research, Norwegian University of Science and Technology.

CASS \& ProClim- (1997). Forschung zu Nachhaltigkeit und Globalem Wandel - Wissenschaftspolitische Visionen der Schweizer Forschenden. Bern: ProClim-/SANW. https:// naturwissenschaften.ch/service/publications/75640-visionen-der-forschenden. Zugegriffen am 21.01.2018.

Defila, R., \& Di Giulio, A. (2018a). Eine Reflexion über Legitimation, Partizipation und Intervention im Kontext transdisziplinärer Forschung. In M. Ukowitz \& R. Hübner (Hrsg.), Partizipation und Intervention. Wege der Vermittlung in der transdisziplinären Forschung. Interventionsforschung, Bd. 3. Wiesbaden: Springer VS. (im Druck).

Defila, R., \& Di Giulio, A. (2018b). What is it good for? Reflecting and systematizing accompanying research to research programs. GAIA, 27 (S1), (S. 97-104). (im Druck).

Defila, R., \& Di Giulio, A. (1998). Interdisziplinarität und Disziplinarität. In J.-H. Olbertz (Hrsg.), Zwischen den Fächern - über den Dingen? Universalisierung versus Spezialisierung akademischer Bildung (S. 111-137). Opladen: Leske \& Budrich. doi: 10.1007/978-3-322-90935-0_6.

Defila, R., Di Giulio, A., \& Scheuermann, M. (2006). Forschungsverbundmanagement. Handbuch für die Gestaltung inter- und transdisziplinärer Projekte. Zürich: vdf Hochschulverlag an der ETH Zürich.

GAIA Special Issue (2018). Labs in the real world: Advancing Transdisciplinarity and Transformations. GAIA, 27 (S1). (im Druck).

Grunwald, A. (2015). Transformative Wissenschaft - eine neue Ordnung im Wissenschaftsbetrieb? GAIA, 24 (1), (S. 17-20).

Klein, J. T. (2014). Interdisciplinarity and Transdisciplinarity: Keyword Meanings for Collaboration Science and Translational Medicine. Journal of Translational Medicine \& Epidemiology, 2 (2), (S. 1024-30).

Krause, N. (2017). Wissenschaft und Gesellschaft - Welche Funktionen erfüllt die Wissenschaft für die Gesellschaft? In H. Kauhaus \& N. Krause (Hrsg.), Fundiert forschen, Wissenschaftliche Bildung für Promovierende und Postdocs (S. 119-149). Wiesbaden: Springer VS.

Krohn, W., Grunwald, A., \& Ukowitz, M. (2017). Transdisziplinäre Forschung revisited. Erkenntnisinteresse, Forschungsgegenstände, Wissensform und Methodologie. GAIA 26 (4), (S. 341-347). doi: 10.14512/gaia.26.4.11. 
Mielke, J., Vermeßen, H., Ellenbeck, S., \& Fernandez Milan, B. (2016). Stakeholder Involvement in sustainability science - a critical view. Energy Research \& Social Science (ERSS), 17, (S. 71-81). doi: 10.1016/j.erss.2016.04.001.

Mobjörk, M. (2010). Consulting versus participatory transdisciplinarity: A refined classification of transdisciplinary research. Futures, 42 (8), (S. 866-873). doi: 10.1016/ j.futures.2010.03.003.

Otero, I., Niewöhner, J., Krueger, T., Doğmus, Ö. C., Himmelreich, J., Sichau, C., \& Hostert, P. (2017). The position of scientists in transformations of human-environment systems. An inquiry into IRI THESys research practices. THESys Discussion paper No. 2017-1. Berlin: Humboldt-Universität zu Berlin.

Rohe, W. (2015). Vom Nutzen der Wissenschaft für die Gesellschaft - Eine Kritik zum Anspruch der transformativen Wissenschaft. GAIA, 24 (3), (S. 156-159).

Schäpke, N., Stelzer, F., Bergmann, M., \& Lang, D. J. (2016). Tentative theses on transformative research in real-world laboratories: First insights from the accompanying research ForReal. Technikfolgenabschätzung - Theorie und Praxis, 25 (3), (S. 10-15).

Schäpke, N., Stelzer, F., Bergmann, M., Singer-Brodowski, M., Wanner, M., Caniglia G., \& Lang, D. (2017). Reallabore im Kontext transformativer Forschung. Ansatzpunkte zur Konzeption und Einbettung in den internationalen Forschungsstand. (No. 1/2017). Leuphana Universität Lüneburg, Institut für Ethik und Transdisziplinäre Nachhaltigkeitsforschung.

Schneidewind, U. (2015). Transformative Wissenschaft - Motor für gute Wissenschaft und lebendige Demokratie. GAIA, 24 (2), (S. 88-91). doi: 10.14512/gaia.24.2.5.

Schneidewind, U., \& Singer-Brodowski, M. (2014). Transformative Wissenschaft. Klimawandel im deutschen Wissenschafts- und Hochschulsystem. 2. Aufl., Marburg: Metropolis.

Scholz, R. (2017). The Normative Dimension in Transdisciplinarity, Transition Management, and Transformation Sciences: New Roles of Science and Universities in Sustainable Transitioning. Sustainability, 9. (S. 991). doi: 10.3390/su9060991.

Strohschneider, P. (2014). Zur Politik der Transformativen Wissenschaft. In A. Brodocz, D. Herrmann, R. Schmidt, D. Schulz, \& J. Schulze Wessel (Hrsg.), Die Verfassung des Politischen (S. 175-192). Wiesbaden: Springer Fachmedien. doi: 10.1007/978-3-65804784-9_10.

TATuP-Schwerpunkt (2016). Reallabore als Orte der Nachhaltigkeitsforschung und Transformation. Technikfolgenabschätzung - Theorie und Praxis, 25 (3), (S. 4-51).

von Unger, H. (2014). Partizipative Forschung. Einführung in die Forschungspraxis. Wiesbaden: Springer VS.

WBGU (Wissenschaftlicher Beirat für Globale Umweltveränderungen) (2011). Welt im Wandel. Gesellschaftsvertrag für eine Große Transformation. Hauptgutachten 2011. Berlin: WBGU. Verfügbar unter: http://www.wbgu.de/hauptgutachten/hg-2011-Trans formation. Zugegriffen am 18.02.2018. 


\section{Beiträge in diesem Buch}

Alcántara, S., Arnold, A., Lindner, D., Busch, S., Dietz, R., Friedrich, M., Ritz, C., \& Sonnberger, M. (2018a). Zwischen Wunsch und Wirkung - Ein transdisziplinärer Visionsworkshop mit Bürgerinnen und Bürgern. In R. Defila \& A. Di Giulio (Hrsg.), Transdisziplinär und transformativ forschen. Eine Methodensammlung (S. 269-299). Wiesbaden: Springer VS.

Alcántara, S., Quint, A., \& Seebacher, A. (2018b). Der Partizipationsmythos „Partizipation in Reallaboren muss repräsentativ sein“. In R. Defila \& A. Di Giulio (Hrsg.), Transdisziplinär und transformativ forschen. Eine Methodensammlung (S. 137-141). Wiesbaden: Springer VS.

Arnold, A., \& Piontek, F. M. (2018). Zentrale Begriffe im Kontext der Reallaborforschung. In R. Defila \& A. Di Giulio (Hrsg.), Transdisziplinär und transformativ forschen. Eine Methodensammlung (S. 143-154). Wiesbaden: Springer VS.

Bachinger, M., Bleher, D., Rau, H., \& Prieß, R. (2018). Die „WiNo-Methode“ zur Identifikation von Wissen in transdisziplinären Netzwerken mithilfe der Wissensbilanzierung. In R. Defila \& A. Di Giulio (Hrsg.), Transdisziplinär und transformativ forschen. Eine Methodensammlung (S. 301-327). Wiesbaden: Springer VS.

Beecroft, R., Trenks, H., Rhodius, R., Benighaus, C., \& Parodi, O. (2018). Reallabore als Rahmen transformativer und transdisziplinärer Forschung: Ziele und Designprinzipien. In R. Defila \& A. Di Giulio (Hrsg.), Transdisziplinär und transformativ forschen. Eine Methodensammlung (S. 75-100). Wiesbaden: Springer VS.

Defila, R., \& Di Giulio, A. (2018c). Partizipative Wissenserzeugung und Wissenschaftlichkeit - ein methodologischer Beitrag. In R. Defila \& A. Di Giulio (Hrsg.), Transdisziplinär und transformativ forschen. Eine Methodensammlung (S. 39-67). Wiesbaden: Springer VS.

Eckart, J., Ley, A., Häußler, E., \& Erl, Th. (2018). Leitfragen für die Gestaltung von Partizipationsprozessen in Reallaboren. In R. Defila \& A. Di Giulio (Hrsg.), Transdisziplinär und transformativ forschen. Eine Methodensammlung (S. 105-135). Wiesbaden: Springer VS.

Puttrowait, E., Dietz, R., Gantert, M., \& Heynold, J. (2018). Der Weg zum Realexperiment - Schlüsselakteure identifizieren, Kooperationsstrukturen aufbauen, Projektideen auswählen. In R. Defila \& A. Di Giulio (Hrsg.), Transdisziplinär und transformativ forschen. Eine Methodensammlung (S. 195-232). Wiesbaden: Springer VS.

Quint, A., Alcántara, S., \& Seebacher, A. (2018). Der Partizipationsmythos „Partizipation in Reallaboren ist per se transparent und muss es auch sein“. In R. Defila \& A. Di Giulio (Hrsg.), Transdisziplinär und transformativ forschen. Eine Methodensammlung (S. 69-73). Wiesbaden: Springer VS.

Rhodius, R., \& Pregernig, M. (2018). Per „Wissensmesse“ zum Forschungsprogramm Arbeitsphasen und Abstimmungsprozesse zwischen Wissenschaft und Praxis. In R. Defila \& A. Di Giulio (Hrsg.), Transdisziplinär und transformativ forschen. Eine Methodensammlung (S. 163-194). Wiesbaden: Springer VS. 
Seebacher, A., Alcántara, S., \& Quint, A. (2018a). Akteure in Reallaboren - Reallabore als Akteure. In R. Defila \& A. Di Giulio (Hrsg.), Transdisziplinär und transformativ forschen. Eine Methodensammlung (S. 155-159). Wiesbaden: Springer VS.

Seebacher, A., Alcántara, S., \& Quint, A. (2018b). Der Partizipationsmythos „Partizipation bedeutet, alle immer an allem zu beteiligen“. In R. Defila \& A. Di Giulio (Hrsg.), Transdisziplinär und transformativ forschen. Eine Methodensammlung (S. 101-104). Wiesbaden: Springer VS.

Trenks, H., Waitz, C., Meyer-Soylu, S., \& Parodi, O. (2018). Mit einer Realexperimentreihe Impulse für soziale Innovationen setzen - Realexperimente initiieren, begleiten und beforschen. In R. Defila \& A. Di Giulio (Hrsg.), Transdisziplinär und transformativ forschen. Eine Methodensammlung (S. 233-268). Wiesbaden: Springer VS.

West, C. (2018): „Wissen to Go“ - Transdisziplinär-transformative Lehre als „Reallabor im Kleinen”. In R. Defila \& A. Di Giulio (Hrsg.), Transdisziplinär und transformativ forschen. Eine Methodensammlung (S. 329-373). Wiesbaden: Springer VS.

Open Access Dieses Kapitel wird unter der Creative Commons Namensnennung 4.0 International Lizenz (http://creativecommons.org/licenses/by/4.0/deed.de) veröffentlicht, welche die Nutzung, Vervielfältigung, Bearbeitung, Verbreitung und Wiedergabe in jeglichem Medium und Format erlaubt, sofern Sie den/die ursprünglichen Autor(en) und die Quelle ordnungsgemäß nennen, einen Link zur Creative Commons Lizenz beifügen und angeben, ob Änderungen vorgenommen wurden.

Die in diesem Kapitel enthaltenen Bilder und sonstiges Drittmaterial unterliegen ebenfalls der genannten Creative Commons Lizenz, sofern sich aus der Abbildungslegende nichts anderes ergibt. Sofern das betreffende Material nicht unter der genannten Creative Commons Lizenz steht und die betreffende Handlung nicht nach gesetzlichen Vorschriften erlaubt ist, ist für die oben aufgeführten Weiterverwendungen des Materials die Einwilligung des jeweiligen Rechteinhabers einzuholen. 